\title{
Relay selection for DF-based cooperative vehicular systems
}

\author{
Ayse Ipek Akin ${ }^{1 *}$, Haci Ilhan ${ }^{1}$ and Özgür Özdemir
}

\begin{abstract}
In this paper, error performance analysis of decode-and-forward (DF)-based cooperative vehicular networks with relay selection is investigated. All of the links in the system are modeled as cascaded Nakagami-m distributed random variables which provide a realistic description of an intervehicular channel. We employ virtual-noise (VN)-based demodulation, maximum likelihood (ML) demodulation, cooperative maximal ratio combining (C-MRC), and log-likelihood-ratio-based transmissions in mitigating error propagation effect encountered in DF cooperative networks. In order to obtain more effective solutions and improve the system performance, we introduce hybrid anti-error propagation approaches, namely VN-MRC, VN-ML, and VN-LLR in which relay selection criterion is derived by using conditional bit error rate (BER) expressions of $\mathrm{VN}$ technique while data detection is performed by utilizing maximal ratio combining (MRC), ML detection, C-MRC and LLR transmission methods. The performance analyses and the numerical results for cascaded Nakagami-m channels have shown that VN-LLR approach provides significant performance improvement with respect to the other considered techniques in DF cooperative vehicular systems with relay selection.
\end{abstract}

Keywords: Cooperative communication; Decode and forward; Relay selection; Maximal ratio combining; Virtual noise; Maximum likelihood; Log-likelihood ratio; Cooperative maximal ratio combining; Cascaded Nakagami-m fading

\section{Introduction}

In most of the wireless applications, the users may not be able to support multiple antennas due to the size, complexity, and power limitations. The fundamental challenges of wireless medium such as time-varying characteristics of the channels and multiuser interference can be mitigated with cooperative diversity which depends on the concept of cooperation among distributed single-antenna wireless nodes and emerged recently as an alternative to multi-antenna systems to obtain spatial diversity [1-3]. The main idea behind cooperative diversity systems is that the source and the destination terminals communicate with each other with the help of intermediate nodes called relay terminals. Various cooperative relaying schemes exist in literature and two of the most well-known are amplify-and-forward (AF) and decode-and-forward (DF) protocols. In AF relaying, the relay terminal transmits a scaled version of received

\footnotetext{
* Correspondence: a.jpekakin@gmail.com

${ }^{1}$ Department of Electronics and Communications Engineering, Yildiz

Technical University, Istanbul 34220, Turkey

Full list of author information is available at the end of the article
}

signal without decoding the message. In DF relaying, the relay terminal decodes its received signal and then reencodes it for transmission to the destination [1-4]. The fundamental destructive effect encountered in AF-based wireless networks is the re-transmission of the amplified version of the noise terms while the most important problem in DF-based cooperative systems is the error propagation due to the decoding errors at relay terminals which cause reduction in the effective signal-tonoise ratio (SNR) at the destination.

Performance of wireless relay networks can further be improved by selection of cooperating relays $[5,6]$. In most of the relay selection-based cooperative network structures in the literature, the data transmission is realized with single relay selection. Bletsas et al. have proposed a relay selection algorithm for multi-relay systems in [7] where the best relay is chosen for transmission. In [8], the authors propose a relay selection criterion in which the relay with the maximum SNR in relay-to-

\section{穴}

(C) 2015 Akin et al.; licensee Springer. This is an Open Access article distributed under the terms of the Creative Commons Attribution License (http://creativecommons.org/licenses/by/2.0), which permits unrestricted use, distribution, and reproduction in any medium, provided the original work is properly credited. 
destination link cooperates. Relay selection approaches based on scaled harmonic means of the instantaneous SNRs of source-to-relay and relay-to-destination [9] links also exist in the literature.

In AF cooperative systems, Anghel and Kaveh showed that maximal ratio combining (MRC) is the optimum detection technique at destination [10]. On the other hand, MRC may not supply full diversity gain in DF cooperative systems because of the error propagation. Although, maximum likelihood (ML) technique may be impractical in some scenarios, it was shown in [2] that ML detection achieves full diversity level for single-relay DF-based cooperative systems. Laneman and Wornell proposed a piecewise linear (PL) approximation [11] which removes non-linear behavior of the optimum ML receiver $[11,12]$ and simplifies the mathematical analyses. However, this approach still requires considering all possible symbol detection cases at the relays as well as the destination which increases the complexity of the receiver exponentially with the modulation order and the number of users. In [13], Yi and Kim showed that cooperative maximal ratio combining (C-MRC) technique has nearly the same performance as the optimum ML technique with less complexity and they combined the C-MRC technique with the relay selection. Another recent demodulation technique developed for combating error propagation problem is virtual noise $(\mathrm{VN})$-based detection [14]. This technique depends on the idea of considering the detection errors at the relay as addition of virtual noise at the destination node. This new concept removes the computational complexity encountered in realization of ML-based receivers and provides full diversity advantage. In [14], VN-based demodulation approach has been applied to different cooperative communication scenarios. In [15-20], log-likelihood-ratio (LLR)-based transmission model which is also known as decodeamplify-forward (DAF) protocol or soft information relaying has been proposed as another promising method for alleviating the effect of error propagation. In this approach, the soft estimates of the corresponding transmitted symbols are forwarded in an analog manner to combine the coding gain in DF protocol and the soft representation of the data in AF technique. Here, relay will first soft decode the received signal, amplify the LLR values of each bit at the output of the decoder, and finally transmit them to destination [18].

Experimental results and theoretical analyses demonstrate that classical fading channels, i.e., Rayleigh, Rician, Nakagami, and the related second-order channel statistics proposed originally for a base station-to-mobile link fail to provide an accurate model for intervehicular links $[21,22]$. Instead, cascaded fading channel models have been proposed which provide a more realistic description of vehicle-to-vehicle channels or mobile-to-mobile channels where two or more independent fading processes are assumed to be generated by independent groups of scatterers around the two mobile terminals $[22,23]$.

The cascaded Rayleigh fading channel [24] obtained by the product of $\mathrm{N}$-independent Rayleigh distributed random variables is one of the important forms of cascaded fading models. For $N=2$, it reduces to double Rayleigh fading distribution which has been considered in [25-27]. Afterwards, this model has been extended to the cascaded Nakagami-m channel in [28-31]. Cascaded Rayleigh and cascaded Nakagami-m fading channels are also studied in cooperative communication systems [32] and references there in. Cascaded Weibull fading channel is investigated in [33] and cascaded generalized- $K$ fading channel is further introduced and investigated in [34]. The outage and the SER performance of two-way communication are examined over cascaded Nakagami$\mathrm{m}$ fading channels in [35]. Moreover, the performance of VN-based detection technique for mitigating error propagation in wireless relay systems employing DF protocol is investigated in [36].

In this paper, we consider a DF-based cooperative vehicular communication system model with relay selection where the underling channels between cooperation nodes are modeled as cascaded Nakagami-m distribution. After investigating the error performance of MRC and C-MRC detectors, we introduce VN-MRC-, VNML-, and VN-LLR-based system models with relay selection. The reminder of this paper is organized as follows: Section 2 presents the system and channel model. In Section 3, we focus on the relay selection methods and performance analysis of considered system. In Section 4, the numerical results are presented. Finally, the concluding remarks are given in Section 5.

\section{System and channel model}

We consider intervehicular cooperative communication system with a source (S), a destination (D), and $M$ relay (R) vehicles as shown in Figure 1. Every terminal operates in half-duplex mode and is equipped with a single antenna.

The normalized distances between the source and $k$ th relay, $k$ th relay and destination, and source and destination are represented by $d_{S R_{k}}, d_{R_{k} D}$, and $d_{S D}$, respectively. The complex fading coefficients of $\mathrm{SR}_{k}, \mathrm{R}_{k} \mathrm{D}$, and SD links are denoted by $\alpha_{S R_{k}}, \beta_{\mathrm{R}_{k} \mathrm{D}}$, and $\alpha_{S D}$, respectively. The magnitude of all of the links follow cascaded Nakagami-m distribution which is the product of $N$ Nakagami-m distributions as $\alpha_{\mathrm{SR}_{k}}=\alpha_{\mathrm{SR}_{k}}^{1} \ldots \alpha_{\mathrm{SR}_{k}}^{N}, \quad \beta_{\mathrm{R}_{k} \mathrm{D}}=\beta_{\mathrm{R}_{k} \mathrm{D}}^{1} \cdots$ $\beta_{\mathrm{R}_{k} \mathrm{D}}^{N}, \alpha_{\mathrm{SD}}=\alpha_{\mathrm{SD}}^{1} \ldots \alpha_{\mathrm{SD}}^{N}$ where $N$ is the degree of cascading. The probability density function (PDF) of Nakagami-m distribution is given by [37]: 


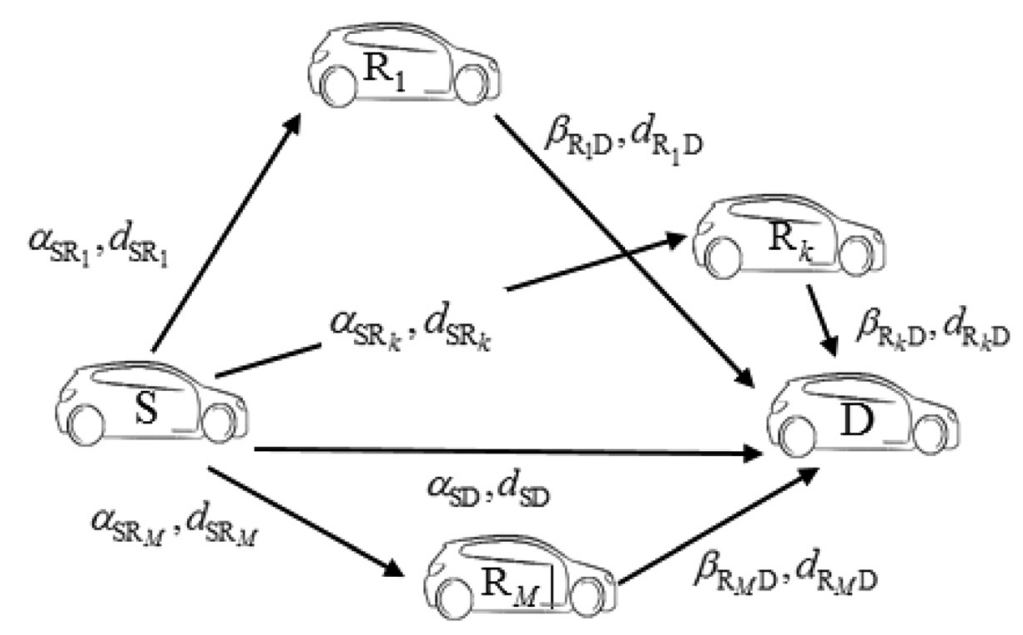

Figure 1 System model.

$$
p_{Z}(z)=\frac{2 m^{m} z^{2 m-1}}{\Gamma(m) \Omega^{m}} \exp \left(-\frac{m z^{2}}{\Omega}\right)
$$

where $Z \in\left\{\alpha_{S D}^{1}, \ldots, \alpha_{S D}^{N}, \alpha_{S R_{k}}^{1}, \ldots, \alpha_{S R_{k}}^{N}, \beta_{R_{k} D}^{1}, \ldots, \beta_{R_{k} D}^{N}\right\}, \quad \Gamma($.$) is the$ Gamma function [37], $\Omega=E\left\{Z^{2}\right\}, m=\Omega / E\left\{Z^{2}-\Omega^{2}\right\} \geq 0.5$ is the Nakagami-m fading parameter and $E\{$.$\} is the stat-$ istical expectation operation. Note that $m=1$ case corresponds to the Rayleigh distribution. By using (1), the PDF of cascaded Nakagami-m fading channel can be given as [28]:

$$
p_{V}(v)=\frac{2}{v \prod_{i=1}^{N} \Gamma\left(m_{i}\right)} G_{0, N}^{N, 0}\left(\left.v^{2} \prod_{i=1}^{N}\left(m_{i} / \Omega_{i}\right)\right|_{m_{1}, \ldots, m_{N}}\right)
$$

where $V \in\left\{\alpha_{\mathrm{SD}}, \quad \alpha_{\mathrm{SR}_{k}}, \quad \beta_{\mathrm{R}_{k} \mathrm{D}}\right\}$ and $G_{. . .}^{. .}($.$) is the Meijer-G$ function [28]. In this paper, destination node is assumed to have full channel state information of the system via pilot signaling, determines the relay with best channel condition and declares the index of the selected relay to all relays through the feedback link.

The transmission is accomplished in two phases. In the first phase, the source transmits its binary phaseshift keying (BPSK) modulation symbol block $x$ and the received signals by the relays and destination can be given as:

$$
\begin{aligned}
& y_{S R_{k}}=\sqrt{E_{s}} x \alpha_{S R_{k}}+n_{S R_{k}}, \\
& y_{S D}=\sqrt{E_{s}} x \alpha_{S D}+n_{S D},
\end{aligned}
$$

where $E_{\mathrm{s}}$ is the average transmitted energy per symbol and $n_{\mathrm{SR}_{k}}$ and $n_{\mathrm{SD}}$ represent noise terms at the $k$ th relay and the destination, respectively. In the second phase, the selected relay terminal estimates the signal received at the first phase and then transmits the estimated symbol block $\hat{x_{k}}$ to the destination. The received signal at the destination can be written as:

$$
y_{R_{k} D}=\sqrt{E_{s}} \hat{x}_{k} \beta_{R_{k} D}+n_{R_{k} D}
$$

where:

$$
\hat{x}_{k}=\arg \min _{x \in\{-1,1\}}\left|y_{S R_{k}}-\sqrt{E_{s}} x \alpha_{S R_{k}}\right|^{2}
$$

and $n_{R_{k} \mathrm{D}}$ is the noise term at the destination. All noise terms in (3) to (5) are the additive white Gaussian noise (AWGN) components modeled as complex random variables with zero mean and variance of $N_{0} / 2$ per dimension. Then, the instantaneous SNRs of the $S \rightarrow R_{k}, R_{k} \rightarrow$ $\mathrm{D}$, and $\mathrm{S} \rightarrow \mathrm{D}$ links can be given as $\gamma_{S R}=E_{S}\left|\alpha_{S R_{k}}\right|^{2} / N_{0}$, $\gamma_{R_{k} D}=E_{s}\left|\beta_{R_{k} D}\right|^{2} / N_{0}$ and $\gamma_{S D}=E_{s}\left|\alpha_{S D}\right|^{2^{k}} / N_{0}$, respectively.

\section{Relay selection criteria and symbol error rate analysis}

In this section, mathematical expression of the relay selection criteria and performance analyses of the considered schemes are provided.

\subsection{MRC-based model}

In MRC-based relaying model, the end-to-end bit error rate (BER) in $\mathrm{S} \rightarrow \mathrm{R}_{k} \rightarrow \mathrm{D}$ link, $P_{\mathrm{eq}}^{\mathrm{b}}\left(\gamma_{\mathrm{SR}_{k}}, \gamma_{\mathrm{R}_{k} \mathrm{D}}\right)$ can be determined by [38]:

$$
\begin{aligned}
P_{e q}^{b}\left(\gamma_{S R_{k}}, \gamma_{R_{k} D}\right)= & {\left[1-P_{S R_{k}}^{b}\left(\gamma_{S R_{k}}\right)\right] P_{R_{k} D}^{b}\left(\gamma_{R_{k} D}\right) } \\
& +\left[1-P_{R_{k} D}^{b}\left(\gamma_{R_{k} D}\right)\right] P_{S R_{k}}^{b}\left(\gamma_{S R_{k}}\right)
\end{aligned}
$$

In (7), $P_{\mathrm{SR}_{k}}^{b}$ and $P_{\mathrm{R}_{k} D}^{b}$ are the BERs of $S \rightarrow \mathrm{R}_{k}$ and $\mathrm{R}_{k} \rightarrow$ D links which can be calculated by $P_{S R_{k}}^{b}=Q\left(\sqrt{2 \gamma_{S R_{k}}}\right)$ 
and $P_{R_{k} D}^{b}=Q\left(\sqrt{2 \gamma_{R_{k} D}}\right)$, respectively, for BPSK modulation where $Q(x)=(1 / \sqrt{2 \pi}) \int_{x}^{\infty} \exp \left(-t^{2} / 2\right) d t$. The equivalent $\mathrm{SNR}$ of $\mathrm{S} \rightarrow \mathrm{R}_{k} \rightarrow \mathrm{D}$ link $\gamma_{\mathrm{eq}, k}$ can be expressed by [38]:

$$
\gamma_{e q, k}=\frac{1}{\eta}\left\{Q^{-1}\left[P_{e q}^{b}\left(\gamma_{S R_{k}}, \gamma_{R_{k} D}\right)\right]\right\}
$$

where $\eta=2$ for BPSK modulation. Therefore, the relay selection criterion for MRC-based system model can be expressed as:

$$
k=\underset{k^{\prime} \in\{1,2, \ldots, M\}}{\operatorname{argmax}} \gamma_{e q, k}
$$

where $k$ denotes the index of the selected relay. At the destination, MRC technique is used to combine the signals $y_{\mathrm{SD}}$ and $y_{\mathrm{R}_{k} \mathrm{D}}$. Thus, the combined signal at the destination can be given as:

$$
y_{D}=\omega_{S D} y_{S D}+\omega_{R_{k} D} y_{R_{k} D}
$$

Here, $\omega_{S D}$ and $\omega_{R_{k} D}$ are the weighting coefficients which are the functions of $\alpha_{\mathrm{SD}}$ and $\beta_{\mathrm{R}_{k} \mathrm{D}}$. Substituting $\omega_{S D}=\alpha_{S D}^{*}$ and $\omega_{R_{k} D}=\beta_{R_{k} D}^{*}$ into (10), the combined signal at the destination can be given as:

$$
y_{D}=\alpha_{S D}^{*} y_{S D}+\beta_{R_{k} D}^{*} y_{R_{k} D}
$$

where (.)* denotes the complex conjugate operator. The received SNR at the destination can be expressed as $\gamma_{t o t}=\gamma_{b}+\gamma_{\mathrm{SD}}$ where $\gamma_{b}=\gamma_{e q, k}$ and the PDF of $\gamma_{t o t}$ can be calculated by:

$$
p\left(\gamma_{t o t}\right)=\int_{0}^{\gamma_{t o t}} p_{\gamma_{S D}}(x) p_{\gamma_{b}}\left(\gamma_{t o t}-x\right) d x
$$
[39]:

The end-to-end probability of error can be written as

$$
P_{e}=P_{\text {prop }} P_{S R_{k}}+\left(1-P_{S R_{k}}\right) P_{M R C},
$$

where $P_{\text {prop }}$ denotes the probability of error propagation and $P_{M R C}$ is the error probability of combined signals. $P_{\text {prop }}$ can be approximated for BPSK modulation by [39]:

$$
P_{\text {prop }} \approx \bar{\gamma}_{b} /\left(\bar{\gamma}_{b}+\bar{\gamma}_{S D}\right)
$$

where $\bar{\gamma}_{b}$ and $\bar{\gamma}_{S D}$ are the expected values of $\gamma_{b}$ and $\gamma_{S D}$, respectively. Similarly, $P_{S R_{k}}$ is the probability of error in the $\mathrm{S} \rightarrow \mathrm{R}_{k}$ link which can be given by:

$$
P_{S R_{k}}=\int_{0}^{\infty} \frac{1}{2} \operatorname{erfc}(\sqrt{x}) p_{\gamma_{S R_{k}}}(x) d x
$$

Here, $p_{\gamma_{S R_{k}}}(\cdot)$ is the PDF of $\gamma_{S R_{k}}$ and $\operatorname{erfc}($.$) is comple-$ mentary error function. $p_{\gamma_{S R_{k}}}(x)$ can be given as [28]:

$$
p_{\gamma_{\mathrm{SR}_{\mathrm{k}}}}(x)=\frac{1}{x \prod_{i=1}^{N} \Gamma\left(m_{i}\right)} G_{0, N}^{N, 0}\left(\left.\frac{x}{\bar{\gamma}_{\mathrm{SR}_{k}}} \prod_{i=1}^{N} m_{i}\right|_{m_{1}, \ldots, m_{N}}\right),
$$

where $\bar{\gamma}_{S R_{k}}$ is $\bar{\gamma}_{S R_{k}}=\left(E_{s} / N_{0}\right) \prod_{i=1}^{N} m_{i}$. By using:

$$
\operatorname{erfc}(x)=\frac{1}{\sqrt{\pi}} G_{1,2}^{2,0}\left(\left.x\right|_{0,0.5} ^{1}\right)^{i=1}
$$

with the help of [40] (Eq. 07.34.21.0011.01), the expression (15) can be calculated as:

$$
P_{\mathrm{SR}_{k}}=\frac{1}{2 \sqrt{\pi} \prod_{i=1}^{N} \Gamma\left(m_{i}\right)} G_{2, N+1}^{N, 2}\left(\left.\frac{1}{\bar{\gamma}_{\mathrm{SR}_{k}}} \prod_{i=1}^{N} m_{i}\right|_{m_{1}, \ldots m_{N}, 0} ^{1,0.5}\right)
$$

The error probability of combined source-destination and relay-destination signals $P_{\mathrm{MRC}}$ can be expressed as [39]:

$$
P_{M R C}=\int_{0}^{\infty} \frac{1}{2} \operatorname{erfc}\left(\sqrt{\gamma_{t o t}}\right) p\left(\gamma_{t o t}\right) d \gamma_{t o t}
$$

The error probability of the considered system can be obtained by substituting (14), (18), and (19) into (13).

\subsection{C-MRC-based model}

The decision rule at the destination for C-MRC-based model can be written as [38]:

$\hat{x}=\arg \min _{x \in A_{x}}\left|\omega_{S D} y_{S D}+\omega_{R_{k} D} y_{R_{k} D}-\left(\omega_{S D} \alpha_{S D}+\omega_{R_{k} D} \beta_{R_{k} D}\right) x\right|^{2}$

where $A_{x}=\{-1,+1\}$ for BPSK modulation. In the previous section, it is mentioned that, in the MRC technique, weighting coefficients were chosen as $\omega_{S D}=\alpha_{S D}^{*}$ and $\omega_{R_{k} D}=\beta_{R_{k} D}^{*}$ to maximize the SNR at the output of combiner. This choice may decrease the average SNR obtained at the destination especially when detection errors occur at the relay terminal. In order to solve this problem C-MRC technique is proposed in which $\omega_{S D}$ is fixed to $\alpha_{S D}^{*}$, while $\omega_{\mathrm{R}_{k} \mathrm{D}}$ is determined by [38]:

$$
\omega_{R_{k} D}\left(\alpha_{S R_{k}}, \beta_{R_{k} D}\right)=\left(\gamma_{e q, k} / \gamma_{R_{k} D}\right) \beta_{R_{k} D}^{*}
$$

In (21), $\gamma_{e q, k}$ is the equivalent SNR given in (8).

Note that in high SNR regime, we obtain $\gamma_{e q} \approx \gamma_{\text {min }}$ where $\gamma_{\min }=\min \left\{\gamma_{\mathrm{R}_{k} \mathrm{D}}, \gamma_{\mathrm{SR}_{k}}\right\}$. And finally, the best relay can be chosen by the help of (9).

\subsection{VN-MRC model}

The main idea of VN-based model is the introduction of virtual noise component at the destination. In this 
approach, detection errors at the relay are modeled as the addition of virtual noise [14]. In this case, we rewrite the received signal at the destination in the second phase as:

$$
\begin{aligned}
y_{R_{k} D} & =\sqrt{E_{s}} \hat{x} \beta_{R_{k} D}+n_{R_{k} D} \\
& =\sqrt{E_{s}} x \beta_{R_{k} D}+\sqrt{E_{s}} e_{r} \beta_{R_{k} D}+n_{R_{k} D} \\
& =\sqrt{E_{s}} x \beta_{R_{k} D}+n_{v}+n_{R_{k} D},
\end{aligned}
$$

where $n_{\mathrm{v}}$ is the virtual noise which is independent from additive white Gaussian noise $n_{R_{k} D}$ and modeled by a zero-mean complex random variable. The variance of term $e_{r}$ in (22) is $N_{0} /\left|\alpha_{S R_{k}}\right|^{2}$ [14]. The combined signal at the destination in case of $\mathrm{VN}$-based detection can be given as [14]:

$$
y_{D}=\omega_{S D} y_{S D}+\omega_{R_{k} D} y_{R_{k} D}
$$

where the weighting coefficients are determined by:

$$
\begin{aligned}
& \omega_{S D}=\alpha_{S D}^{*} / N_{0} \\
& \omega_{R_{k} D}=\beta_{R_{k} D}^{*} / N_{0}\left(1+\left|\beta_{R_{k} D}\right|^{2} /\left|\alpha_{S R_{k}}\right|^{2}\right) .
\end{aligned}
$$

Note that this combining approach eliminates the decoding complexity in the classical ML-based detectors that increases exponentially with the number of the components in the signal constellation. The conditional $\mathrm{BER}$ of the proposed system for a given relay in $\mathrm{VN}$ with MRC-based model can be expressed as:

$$
\begin{aligned}
P_{k}= & \left(1-Q\left(\sqrt{2 \gamma_{S R}}\right)\right) Q\left(\sqrt{\frac{2\left(\gamma_{S D}+\gamma_{E Q}\right)^{2}}{\gamma_{S D}+\gamma_{E Q}^{\prime}}}\right) \\
& +Q\left(\sqrt{2 \gamma_{S R}}\right) Q\left(\sqrt{\frac{2\left(\gamma_{S D}-\gamma_{E Q}\right)^{2}}{\gamma_{S D}+\gamma_{E Q}^{\prime}}}\right)
\end{aligned}
$$

where:

$$
\begin{aligned}
& \gamma_{E Q}=\frac{\left|\alpha_{S R_{k}}\right|^{2}\left|\beta_{R_{k} D}\right|^{2}}{\left(\left|\alpha_{S R_{k}}\right|^{2}+\left|\beta_{R_{k} D}\right|^{2}\right) N_{0}}, \\
& \gamma_{E Q}^{\prime}=\frac{\left|\alpha_{S R_{k}}\right|^{4}\left|\beta_{R_{k} D}\right|^{2}}{\left(\left|\alpha_{S R_{k}}\right|^{2}+\left|\beta_{R_{k} D}\right|^{2}\right) N_{0}} .
\end{aligned}
$$

The conditional BER expression given in (26) can be used as the best relay selection criterion which can be given by:

$$
k=\underset{k^{\prime} \in\{1,2, \ldots, M\}}{\arg \max } P_{k^{\prime}} .
$$

Note that this selection criterion in (28) will also be employed in VN-ML and VN-LLR models given in the following sections.

\subsection{VN-ML model}

After choosing the best relay with the help of criterion in (28), ML technique can be used at the destination for detection. In [11,12], LLR of the ML detection for uncoded DF cooperation with binary modulations is given as:

$$
\operatorname{LLR}=t_{0}+\psi\left(t_{1}\right)
$$

where:

$$
\psi\left(t_{1}\right)=\ln \frac{\left(1-\varepsilon_{r}\right) e^{t_{1}}+\varepsilon_{r}}{\varepsilon_{r} e^{t_{1}}+\left(1-\varepsilon_{r}\right)} .
$$

Here, $\varepsilon_{r}$ is the average BER at the $k$ th relay, and it can be calculated for BPSK signaling as [41]:

$$
\varepsilon_{r}=Q\left(\sqrt{\frac{2 E_{s}}{N_{0}}}\left|\alpha_{S R_{k}}\right|\right),
$$

$t_{0}$ and $t_{1}$ are log-likelihood ratios of direct and relay links, respectively, for BPSK modulation, which can be given by [41]:

$$
\begin{aligned}
t_{0}= & \frac{4 \sqrt{E_{s}} \Re\left\{y_{S D} \alpha_{S D}^{*}\right\}}{N_{0}} \\
t_{1}= & \frac{4 \sqrt{E_{s}} \Re\left\{y_{R_{k} D} \beta_{R_{k} D}^{*}\right\}}{N_{0}} .
\end{aligned}
$$

As mentioned before, in some scenarios, performance analysis of ML technique can be really hard because of the nonlinear behavior of the ML detection given in (29). To overcome this problem, an alternative detector is proposed in [12] which is called as detector with piecewise-linear (PL) combiner. This approximation can be expressed as:

$$
\psi\left(t_{1}\right) \approx \psi_{P L}\left(t_{1}\right)=\left\{\begin{array}{cr}
T_{1}, & t_{1} \geq T_{1} \\
t_{1}, & -T_{1}<t_{1}<T_{1} \\
-T_{1}, & t_{1} \leq-T_{1}
\end{array}\right\}
$$

where $T_{1}=\ln \left[\left(1-\varepsilon_{r}\right) / \varepsilon_{r}\right]$ and $\varepsilon_{r}<1 / 2$.

\subsection{VN-LLR model}

In this approach, after the relay selection is realized by using Equation 28, the soft information is transmitted to the destination by amplifying (scaling) and forwarding the LLRs at the output of the decoder (decoder-LLR) [17] instead of making binary hard decisions.

After choosing the best relay with the help of (28), the signal at the relay is given as [18]:

$$
x_{R}(i)=l_{R} E_{L L R}, \quad i=1, \ldots, Q
$$

where $Q$ is the length of source packet, $l_{R}$ is the decoder-LLR at the relay and $E_{\mathrm{LLR}}$ is the energy of $l_{R} . l_{R}$ and $E_{\mathrm{LLR}}$ can be calculated as: 


$$
\begin{aligned}
& l_{R}=\frac{4 \sqrt{E_{s}}}{N_{0}} \Re\left\{y_{S R_{k}} \alpha_{S R_{k}}\right\}, \\
& E_{L L R}=\frac{E_{s} Q}{\sum_{i=1}^{Q}\left|l_{R}\right|^{2}} .
\end{aligned}
$$

In the second phase, the relay terminal transmits LLR information $l_{\mathrm{R}}$ to the destination. Thus, the received signal at the destination can be expressed as:

$$
y_{R_{k} D}=\sqrt{E_{L L R}} l_{R} \beta_{R_{k} D}+n_{R_{k} D}
$$

where $n_{\mathrm{R}_{k} \mathrm{D}}$ is the noise term which is AWGN component modeled as complex random variable with zero mean and variance of $N_{0} / 2$ per dimension. Finally, the optimal signal combination at the destination is expressed as:

$$
l_{\text {coop }}=l_{S D}+l_{R_{k} D}
$$

where $l_{S D}$ and $l_{R_{k} D}$ are the LLRs of source-destination and $k$ th relay-destination channels, respectively, and can be calculated by using [17] as:

$$
l_{R_{k} D}=\frac{16 E_{s}\left|\alpha_{S R_{k}}\right|^{2}\left|\beta_{R_{k} D}\right|^{2} E_{L L R}}{16 E_{s}\left|\alpha_{S R_{k}}\right|^{2}\left|\beta_{R_{k} D}\right|^{2} E_{L L R}^{2}+N_{0}^{2}\left|\beta_{R_{k} D}\right|^{2}} \Re\left\{y_{R_{k} D} \beta_{R_{k} D}^{*}\right\}
$$

$$
l_{S D}=\frac{4 \sqrt{E_{s}}}{N_{0}} \Re\left\{y_{S D} \alpha_{S D}^{*}\right\}
$$

\section{Simulation results}

In this section, we present the Monte Carlo simulation results to show the performance of the proposed schemes in intervehicular DF-based cooperative communication system with relay selection. Without the loss of generality, the distance between source and destination is normalized to 1 and error performance of considered system is obtained for different normalized locations of the relays. The links between $S \rightarrow D, S \rightarrow R_{k}$, and $R_{k} \rightarrow$ $\mathrm{D}$ are modeled as cascaded Nakagami-m distribution. The degree of cascading and the fading parameter are chosen as $N=1,2,4$ and $m=2,3$, respectively. The variance of fading coefficient between two terminal $(i, j)$ is determined by using a path-loss model in the form of $\sigma_{i j}^{2}=d_{i j}^{-v}$, and the path loss coefficient is assigned as $v=4$ [12]. BPSK modulation is considered and the number of relays is chosen to be $M=2$ and 3. The SNR comparisons are done at a BER of $10^{-5}$.

In Figure 2, the average BER performance curves of the considered systems in case of $M=3, N=2, m=2$, and $d_{\mathrm{SR}}=0.9$ are presented. It is seen from this figure that VN-LLR-based relay selection scheme provides approximately $3.2,1.7,1.4$, and $1 \mathrm{~dB}$ performance improvements compared to the system models with MRC, VN-MRC, C-MRC, and VN-ML, respectively.

In order to determine the effect of fading parameter on the performance of the system, we propose the numerical results for $M=3, N=2, m=3$, and $d_{\mathrm{SR}}=0.9$ in Figure 3. As seen from this figure, VN-LLR-based relay selection scheme outperforms MRC, VN-MRC, C$\mathrm{MRC}$, and $\mathrm{VN}-\mathrm{ML}$ techniques by about 2.4, 2, 1.6, and

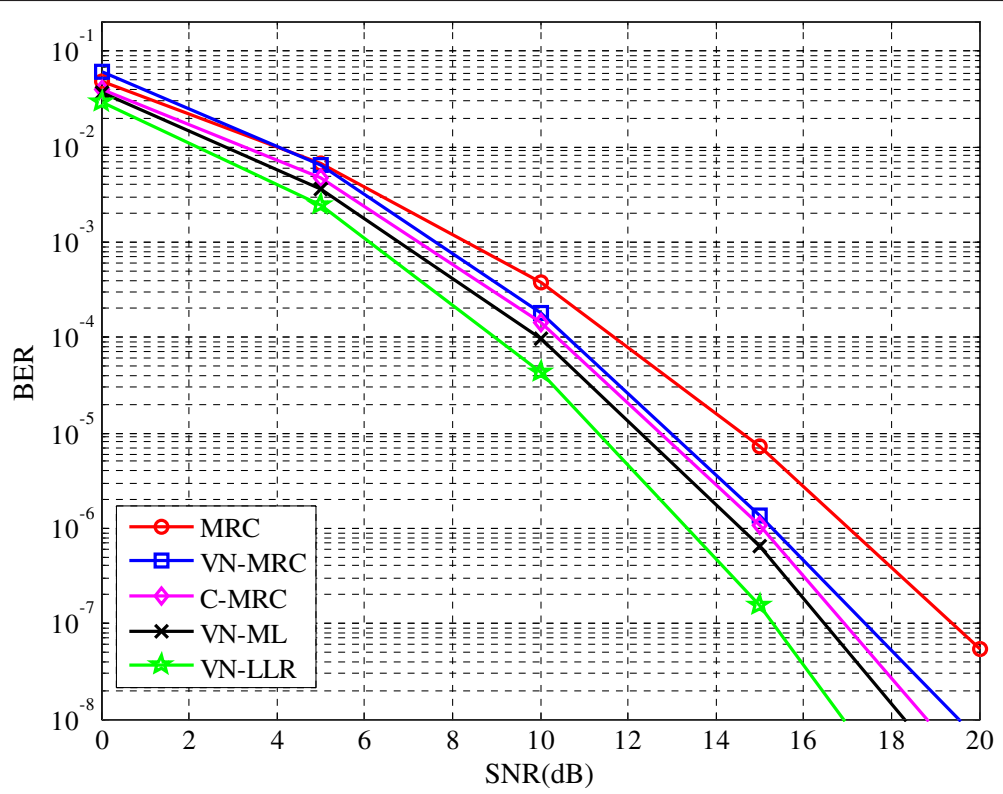

Figure 2 Error performance curves for when the relays are closed to the destination. $M=3, N=2, m=2$, and $d_{\mathrm{SR}}=0.9$. 


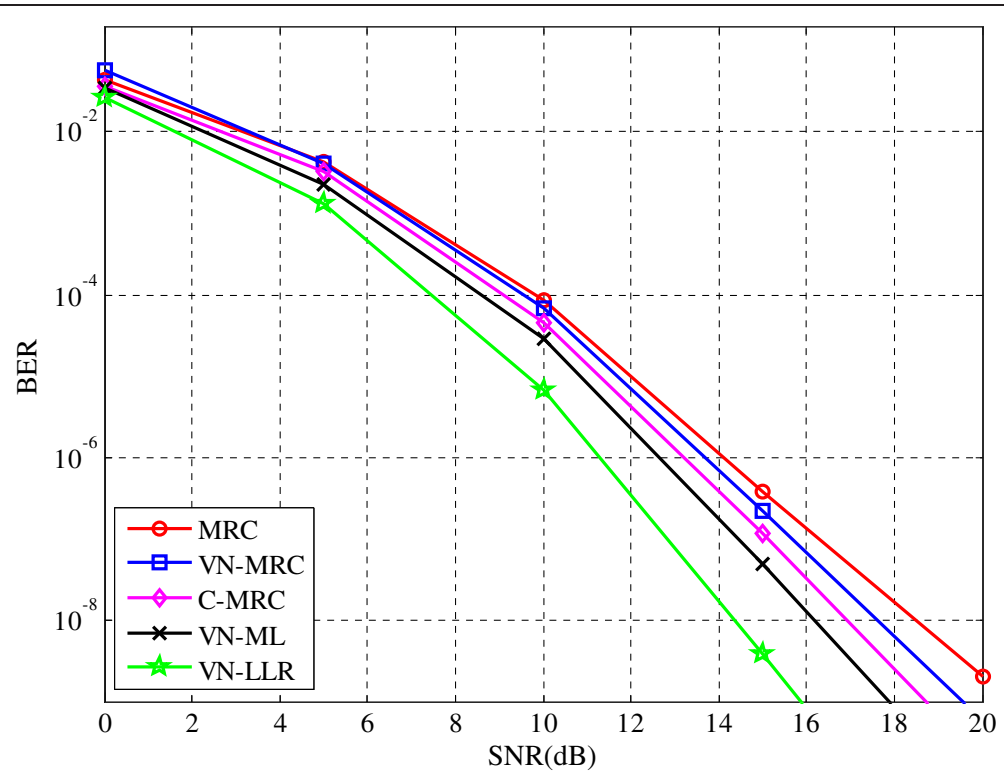

Figure 3 Numerical result for $M=3, N=2, m=3$, and $d_{\mathrm{SR}}=0.9$.

$1.2 \mathrm{~dB}$, respectively. The numerical results for $m=2$ and $m=3$ in Figures 2 and 3 also show that the SNR gains supplied by the $m=3$ case compared to the $m=$ 2 case are approximately $2.6,1.4,1.4,1.5$, and $1.7 \mathrm{~dB}$ for MRC, VN-MRC, C-MRC, VN-ML, and VN-LLR approaches, respectively.

Figure 4 depicts the simulated average BER of MRC, VNMRC, and VN-LLR techniques with relay locations $d_{\mathrm{SR}}=$ 0.1 and 0.9 , degree of cascading $N=2$, fading parameter $m=2$, and number of relays $M=2$. It is seen that when the relay is located closed to destination, i.e., $d_{\mathrm{SR}}=0.9$, an SNR loss occurs with respect to the scheme with $d_{\mathrm{SR}}=0.1$. The amounts of these SNR losses are $0.7 \mathrm{~dB}$ for VNLLR, 2.4 dB for VN-MRC, and $4.5 \mathrm{~dB}$ for MRC. As it can be seen from this figure, the probability of detection error at the relay increases when the distance of SR link increases, since the average SNR of this link decreases. It is also observed from Figure 4 that all of the models give nearly the same average BER performance when the relay is located closed to the source, i.e., $d_{\mathrm{SR}}=0.1$, since the quality of $\mathrm{S} \rightarrow \mathrm{R}$ link is quite well in this channel settings.

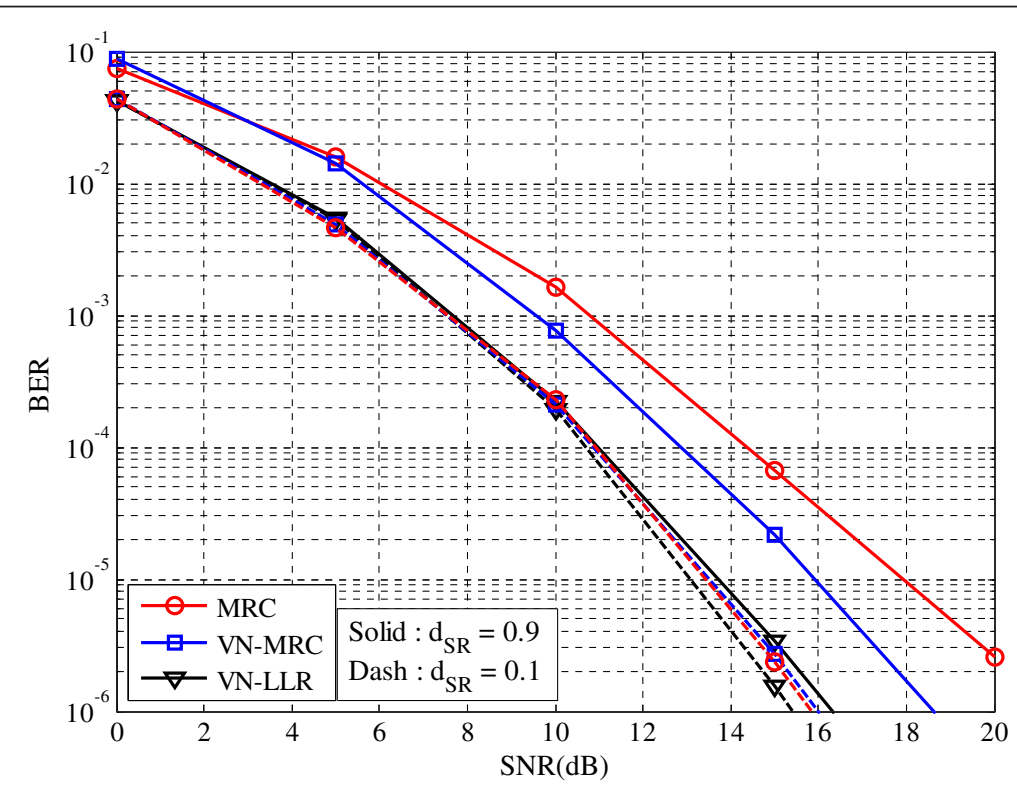

Figure 4 Error performance curves for different relay locations when $m=2, N=2$, and $M=2$. 


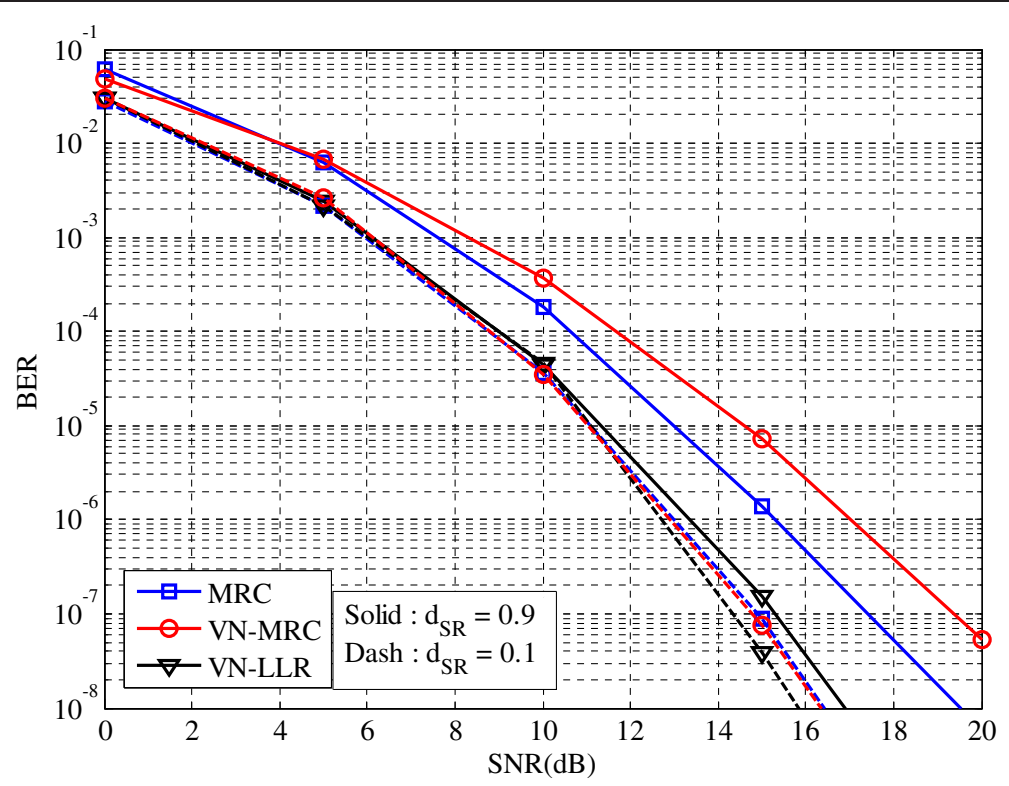

Figure 5 Error performance curves for different relay locations when $m=2, N=2$, and $M=3$.

In Figure 5, the average BER curves of MRC, VNMRC, and VN-LLR techniques with relay locations $d_{\mathrm{SR}}=0.1$ and 0.9 , degree of cascading $N=2$, fading parameter $m=2$, and number of relay $M=3$ are presented. It is seen that the system performance increases as the number of relay increases. The BER performance of the system decreases nearly about 1.8 , 3.5, and $0.2 \mathrm{~dB}$ for MRC, VN-MRC, and VN-LLR techniques, respectively, as the relay terminals gets closer to the destination.

The performance comparison of all of the models for different values of cascading parameter $N$ is demonstrated in Figure 6. It is seen that all approaches provide better average BER performance with decreased degree of cascading $(N)$. The performance improvement with decreased cascading parameter is nearly about $3.4,2.8$, and $2.7 \mathrm{~dB}$ for VN-MRC, VN-ML, and VN-LLR, respectively.

\section{Conclusions}

In this paper, the performance analysis of intervehicular cooperative communication system with relay selection is examined where the links between $\mathrm{S} \rightarrow \mathrm{D}, \mathrm{S} \rightarrow \mathrm{R}_{k}$, and $\mathrm{R}_{k} \rightarrow \mathrm{D}$ have cascaded Nakagami-m distribution. The simulation results are presented when MRC, C-MRC,

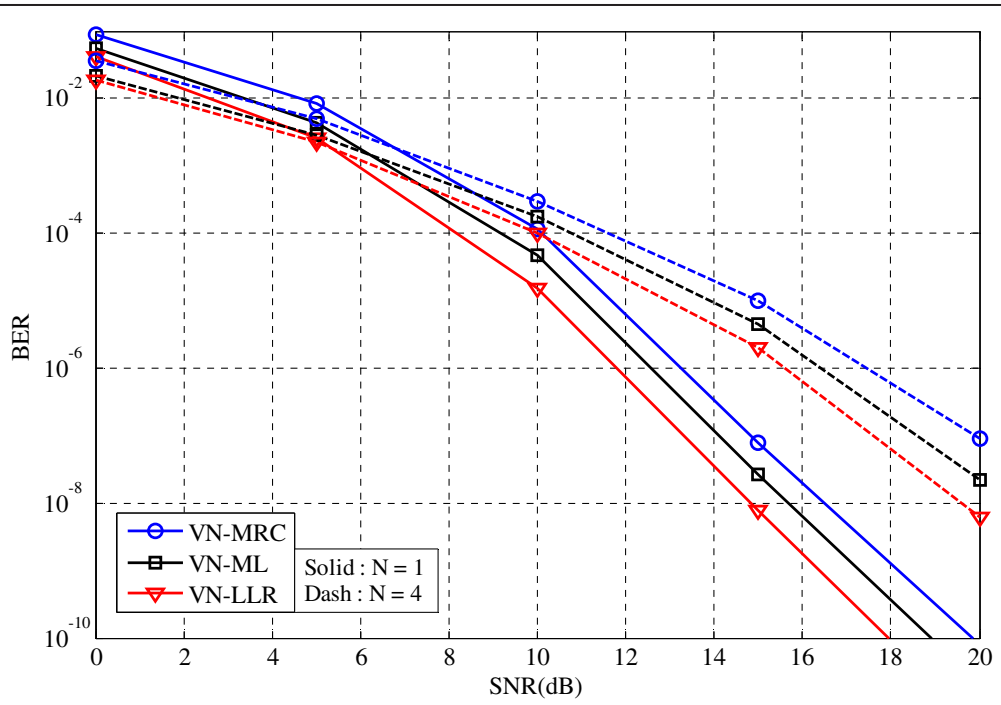

Figure 6 Error performance curves for different values of $\boldsymbol{N}$ when relays are closed to the destination. $m=2, M=3$. 
VN-MRC, VN-ML, and VN-LLR methods are used at destination. One can observe from the results that the performance of considered system increases with decrement of degree of cascading $N$, increment of fading parameter $m$ and number of relay $M$. It is also seen that the best performance is obtained in case of the VN-LLR technique.

\section{Competing interests}

The authors declare that they have no competing interests.

\section{Acknowledgements}

The work of authors are supported by the Scientific and Technological Research Council of Turkey under Project 113E229.

\section{Author details}

'Department of Electronics and Communications Engineering, Yildiz Technical University, Istanbul 34220, Turkey. ${ }^{2}$ Department of Electrical and Electronics Engineering, Selcuk University, Konya 42030, Turkey.

Received: 30 January 2014 Accepted: 6 January 2015

Published online: 18 February 2015

\section{References}

1. J Laneman, D Tse, G Wornell, Cooperative diversity in wireless networks: efficient protocols and outage behaviour. IEEE Trans. Inf. Theory 50(12), 3062-3080 (2002)

2. A Sendonaris, E Erkip, B Aazhang, User cooperation diversity part I: system description. IEEE Trans. Commun. 51(11), 1927-1938 (2003)

3. A Sendonaris, E Erkip, B Aazhang, User cooperation diversity part II: implementation aspects and performance analysis. IEEE Trans. Commun. 51(11), 1939-1948 (2003)

4. A Nosratinia, TE Hunter, Diversity through coded cooperation. IEEE Trans Wireless Commun 5(2), 283-289 (2006)

5. A Adinoyi, Y Fan, H Yanikomeroglu, HV Poor, F Al-Shaalan, Performance of selection relaying and cooperative diversity. IEEE Trans Wireless Commun 8(12), 5790-5794 (2009)

6. GC Alexandropoulos, A Papadogiannis, K Berberidis, Performance analysis of cooperative networks with relay selection over Nakagami-m fading channels. IEEE Signal Process Lett 17(5), 441-444 (2010)

7. A Bletsas, A Khisti, DP Reed, A Lippman, A simple cooperative diversity method based on network path selection. IEEE J Select Areas Commun 24(3), 659-672 (2006)

8. E Beres, R Adve, Selection cooperation in multi-source cooperative networks. IEEE Trans Wireless Commun 7(1), 118-127 (2008)

9. AS Ibrahim, AK Sadek, W Su, KIR Liu, Cooperative communications with relay selection: when to cooperate and whom to cooperate with? IEEE Trans Wireless Commun 7(7), 2814-2827 (2008)

10. PA Anghel, M Kaveh, Exact symbol error probability of a cooperative network in a Rayleigh- fading environment. IEEE Trans Wireless Commun 3(5), 1416-1421 (2004)

11. J Laneman, GW Wornell, Energy-efficient antenna-sharing and relaying for wireless networks (Paper presented at the Proceedings of the IEEE Wireless Communications and Networking Conference, Chicago, 2000)

12. D Chen, J Laneman, Modulation and demodulation for cooperative diversity in wireless systems. IEEE Trans Wireless Commun 5(7), 1785-1794 (2006)

13. Z Yi, IM Kim, Diversity order analysis of the decode-and-forward cooperative networks with relay selection. IEEE Trans Wireless Commun 7(5), 1792-1799 (2008)

14. D Kim, H-M Kim, G-H Im, Improved network-coded cooperative transmission with low-complexity adaptation to wireless channels. IEEE Trans. Commun. 59(10), 2916-2926 (2011)

15. HV Khuong, HY Kong, LLR-based decode-and-forward protocol for relay networks and closed-form BER expressions. IEICE Trans Fundamentals E89-A(6), 1832-1836 (2006)

16. P Weitkemper, D Wübben, V Kühn, K-D Kammeyer, Soft information relaying for wireless networks with error-prone source-relay link (Paper presented at the 7th international ITG conference on source and channel coding (SCC) Ulm, Germany, 2008)
17. X Bao, J Li (Tiffany), Efficient message relaying for wireless user cooperation: decode-amplify-forward (DAF) and hybrid DAF and coded-cooperation. IEEE Trans Wireless Commun 6(11), 3975-3984 (2007)

18. X Bao, J Li, (Tiffany), Decode-amplify-forward (DAF, a new class of forwarding strategy for wireless relay channels (Paper presented at the IEEE 6th workshop on signal processing advances in wireless communications, New York, USA, 2005)

19. S Ibi, N Takada, S Sampei, Bit-wise partial noise elimination in cooperative decode-amplify-forward relay node (Paper presented at the IEEE 75th vehicular technology conference, VTC Spring, Yokohoma, 2012)

20. MA Karim, J Yuan, Z Chen, J Li, Soft information relaying in fading channels. IEEE Wireless Commun Lett 1(3), 233-236 (2012)

21. AS Akki, F Haber, A statistical model of mobile-to-mobile land communication channel. IEEE Trans. Veh. Technol. 35(1), 2-7 (1986)

22. IZ Kovacs, Dissertation, Aalborg University, 2002.

23. Y Chen, GK Karagiannidis, H Lu, N Cao, Novel approximations to the statistics of products of independant random variables and their applications in wireless communications. IEEE Trans. Veh. Technol. 61(2), 443-454 (2012)

24. J Salo, H El-Sallabi, P Vainikainen, The distribution of the product of independent Rayleigh random variables. IEEE Trans. Antennas Propag. 54(2), 639-643 (2006)

25. V Erceg, SJ Fortune, J Ling, A Rustako, R Valenzuela, Comparisons of computer-based propagation prediction tool with experimental data collected in urban microcellular environments. IEEE J Sel Areas Commun 15(4), 677-684 (1997)

26. M Uysal, Maximum achievable diversity order for cascaded Rayleigh fading channels. IET Electronics Lett 41(23), 1289-1290 (2005)

27. Z Tirkan, M Shirkhani, A Taherpour, M Uysal, Performance of cooperative amplify-and-forward protocols in vehicular ad-hoc networks (Paper presented at the international conference on wireless communications and signal Processing (WCSP), Huangshan, 2012)

28. GK Karagiannidis, NC Sagias, PT Mathiopoulos, N*Nakagami: A novel stochastic model for cascaded fading channels. IEEE Trans. Commun. 55(8), 1453-1458 (2007)

29. S Ahmed, LL Yang, L Hanzo, Probability distributions of products of Rayleigh and Nakagami-m variables using Mellin transform (Paper presented at the international conference on communications (ICC), Kyoto, 2011)

30. PM Shankar, Performance of $N^{*}$ Nakagami cascaded fading channels in dual selection combining diversity (Paper presented at the 7th international IEEE wireless communication. Mobile computer conference (IWCMC), Istanbul, 2011)

31. Z Zheng, L Wei, J Hamalainen, Novel approximations to the statistics of general cascaded Nakagami-m channels and their applications in performance analysis (Paper presented at the IEEE international conference on communications (ICC), Budapest, 2013)

32. H Ilhan, M Uysal, I Altunbas, Cooperative diversity for inter-vehicular communication: Performance analysis and optimization. IEEE Trans. Veh. Technol. 58(7), 3301-3310 (2009)

33. NC Sagias, GS Tombras, On the cascaded Weibull fading channel model. J. Frankl. Inst. 344(1), 1-11 (2007)

34. I Trigui, A Laourine, S Affes, A Stephenne, On the performance of cascaded generalized K fading channels (Paper presented at the IEEE global telecommunications conference, GLOBECOM, Honolulu, 2009)

35. H Ilhan, Performance analysis of two-way AF relaying systems over cascaded Nakagami-m fading channels. IEEE Signal Process Lett 19(6), 332-335 (2012)

36. Al Akin, $\mathrm{H}$ llhan, O Ozdemir, Relay selection for decode-and-forward cooperative vehicular networks with virtual noise modeling (Paper presented at the 13th international conference on ITS telecommunications (ITST), Tampere, 2013)

37. MK Simon, MS Alouini, Digital Communication Over Fading Channels, 2nd edn. (Wiley, Hoboken, New Jersey, 2005)

38. T Wang, A Cano, GB Giannakis, High-performance cooperative demodulation with decode-and-forward relays. IEEE Trans. Commun. 55(7), 1427-1438 (2007)

39. K Tourki, M-S Alouini, H-C Yang, Exact performance analysis of decode-andforward opportunistic relaying (Paper presented at the IEEE 11th international workshop on signal processing advances in wireless communications (SPAWC), Marrakech, 2010)

40. Wolfram Research, MeijerG available online at http://functions.wolfram.com (Accessed August 15th, 2013)41

41. A Goldsmith, Wireless Communications (Cambridge University Press, New York, 2005) 